\title{
Maternal peanut consumption and risk of peanut allergy in childhood
}

\author{
Elissa M. Abrams MD, Scott H. Sicherer MD
}

Cite as: CMAJ 2018 July 9;190:E814-5. doi: 10.1503/cmaj.180563

$\mathbf{P}$

eanut allergy in children is on the rise in North America, with a self-reported prevalence of $1.4 \%$ in 2008 , up from $0.4 \%$ in $1997(p<0.001) .{ }^{1}$ As a result, prevention of peanut allergy has become an important public health goal. A recent guideline recommends early introduction of infant-safe forms of peanut protein as a means of prevention, ${ }^{2}$ but evidence regarding peanut consumption in pregnancy and risk of subsequent peanut allergy in offspring is conflicting. Although older studies suggest an increased risk of childhood peanut allergy with maternal peanut ingestion, recent studies suggest that peanut intake in pregnancy may have a preventive effect on development of peanut allergy in children.

Several studies have suggested an increased risk of allergy in childhood following maternal peanut ingestion. For example, a retrospective study involving 503 highly atopic infants, with likely milk or egg allergy, noted in multivariate analysis that maternal peanut consumption twice or more per week in pregnancy was associated with strong peanut sensitization (peanutspecific immunoglobulin $\mathrm{E}[\mathrm{IgE}] \geq 5 \mathrm{kU} / \mathrm{L}$ ) in infancy (odds ratio [OR] 2.9, 95\% confidence interval [Cl] 1.7-4.9). ${ }^{3}$ A case-control study involving 403 infants found that maternal peanut consumption in pregnancy was associated with a significantly higher risk of allergy in infancy compared with age-matched infants with no history of atopic disease (OR 4.22, 95\% $\mathrm{Cl} 1.57-11.30) .{ }^{4}$ In addition, a small retrospective study involving 43 children found that mothers who consumed peanuts more than once a week in pregnancy were significantly more likely to have a peanutsensitized child than mothers who consumed peanuts less than once a week (OR 3.97, 95\% Cl 0.73-24)..$^{5}$

In contrast, other studies have found no link between maternal peanut ingestion and rate of allergy in offspring. For example, a cohort study involving 13971 preschool children found no association between maternal consumption of peanuts and challenge-proven peanut allergy. ${ }^{6}$ Interestingly, this study was also unable to detect peanut-specific IgE in cord blood, suggesting no peanut sensitization in utero. A questionnaire-based, case-control study that compared 133 peanut-allergic children, high-risk controls with egg allergy and controls with no allergy found that maternal peanut ingestion in pregnancy did not increase risk of peanut allergy in childhood. ${ }^{7}$

\section{KEY POINTS}

- The literature about maternal peanut consumption and risk of peanut allergy in childhood is conflicting.

- Guidelines have changed, with more recent guidelines recommending no restriction on maternal diet during pregnancy as a means of allergy prevention.

- A recent guideline recommends early introduction of infant-safe forms of peanut protein as an effective means of peanut allergy prevention.

- Current evidence does not support women avoiding peanuts in pregnancy to prevent peanut allergy, irrespective of maternal atopic status.

- There is insufficient evidence to suggest that women be encouraged to ingest peanut in pregnancy for prevention of peanut allergy.

A systematic review on the influence of early life exposure to peanuts and the subsequent development of peanut sensitization from 2010, based on two case-control studies and one crosssectional study, notes heterogeneous literature that was "limited in quality" (largely owing to a lack of adjustment for possible confounding variables and the predominantly atopic populations studied), which "hindered the development of definitive conclu-

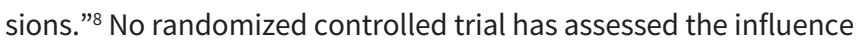
of maternal peanut ingestion on childhood peanut allergy.

Guidelines regarding maternal peanut consumption have changed over time. Although older guidelines recommended that mothers at high risk of having an atopic infant avoid peanut in pregnancy, more recent guidelines such as the 2013 Canadian Paediatric Society guideline on allergy prevention, recommend no restriction on maternal diet during pregnancy as a means of childhood allergy prevention. ${ }^{9}$ The most recent American Academy of Allergy, Asthma and Immunology guideline on prevention of allergic disease notes that, owing to inconclusive data on the role of maternal peanut ingestion, "no recommendation on maternal avoidance of peanut during pregnancy can be made at this time."10

Since the publication of these guidelines, a large prospective national cohort study involving 10901 mothers and their children, published in 2014, documented a decreased risk of childhood 
peanut allergy with maternal peanut consumption in pregnancy. The Growing Up Today Study 2 prospectively followed mothers and their children from peripregnancy until adolescence. ${ }^{11}$ This study found a strong inverse association between peripregnancy peanut and tree nut consumption and risk of peanut and tree nut allergy in childhood. There was a significantly lower incidence of physician-confirmed peanut allergy among children whose mothers consumed more peanuts and tree nuts in their peripregnancy diet (five times or more v. less than once per month; OR $0.31,95 \% \mathrm{Cl}$ 0.13-0.75). In addition, a longitudinal epidemiologic cohort study involving 1277 mother-child pairs unselected for atopy noted reduced peanut allergy in childhood with increased maternal peanut ingestion during the first trimester - each additional z-score of maternal peanut intake was associated with a $47 \%$ reduced odds of peanut allergy in childhood (OR 0.53, 95\% Cl 0.30-0.94)..$^{12}$

There are several possible explanations for the discrepancies in the literature. Most studies suggesting an increased risk with maternal consumption had an outcome of peanut sensitization (i.e., positive peanut-specific IgE testing) instead of peanut allergy, in contrast to the newest literature. In addition, these retrospective studies are limited by recall bias. Other factors (e.g., atopic status, level of antigen exposure, peanut consumption while breastfeeding and environmental exposure) may play a role, and studies cannot dissociate maternal pregnancy diet from the many oral and environmental exposures after birth. Studies have noted environmental peanut exposure in childhood (i.e., household peanut ingestion) to be a risk factor for peanut allergy (in the context of delayed oral exposure). For example, in one study, median household peanut consumption was 10 -fold higher for peanutallergic children than for high-risk controls $(p<0.0001) .^{7}$ This is in keeping with the theory that peanut sensitization occurs via the cutaneous route from environmental exposure in childhood.

Avoiding peanut consumption in pregnancy is not without potential harms. A Cochrane Review on maternal dietary antigen avoidance during pregnancy, though not looking specifically at peanuts, found that antigen avoidance diets were associated with a significantly lower mean gestational weight gain, a nonsignificantly higher risk of preterm birth, and a nonsignificant reduction in mean birth weight, while also noting no significant reduction in atopic disease. ${ }^{13}$

It is likely that other factors (e.g., environmental peanut exposure, maternal peanut consumption while breastfeeding, age of peanut introduction in childhood, maternal diet in general and breastfeeding in general) play a role in peanut sensitization in children.

Although further studies are required to settle the question, current evidence cannot support a recommendation for women to avoid peanuts in pregnancy as a strategy to prevent peanut allergy, irrespective of maternal atopic status. We cannot advise that pregnant women should be encouraged to ingest peanut or suggest an amount of peanut to be ingested to ensure a preventive effect, as there is insufficient evidence to support it at this time.

\section{References}

1. Sicherer SH, Muñoz-Furlong A, Godbold JH, et al. US prevalence of selfreported peanut, tree nut, and sesame allergy: 11-year follow up. J Allergy Clin Immunol 2010;125:1322-6.

2. Togias A, Cooper SF, Acebal ML, et al. Addendum guidelines for the prevention of peanut allergy in the United States: report of the National Institute of Allergy and Infectious Diseases-sponsored expert panel. J Allergy Clin Immunol 2017;139:29-44.

3. Sicherer SH, Wood RA, Stablein D, et al. Maternal consumption of peanut during pregnancy is associated with peanut sensitization in atopic infants. J Allergy Clin Immunol 2010;126:1191-7.

4. DesRoches A, Infante-Rivard C, Paradis L, et al. Peanut allergy: Is maternal transmission of antigens during pregnancy and breastfeeding a risk factor? J Investig Allergol Clin Immunol 2010;20:289-94.

5. Frank L, Marian A, Visser M, et al. Exposure to peanuts in utero and in infancy and the development of sensitization to peanut allergens in young children. Pediatr Allergy Immunol 1999;10:27-32.

6. Lack G, Fox D, Northstone K, et al.; Avon Longitudinal Study of Parents and Children Study Team. Factors associated with the development of peanut allergy in childhood. N Engl J Med 2003;348:977-85.

7. Fox AT, Sasieni $P$, du Toit $G$, et al. Household peanut consumption as a risk factor for the development of peanut allergy. J Allergy Clin Immunol 2009;123:417-23.

8. Thompson RL, Miles LM, Lunn J, et al. Peanut sensitisation and allergy: influence of early life exposure to peanuts. Br J Nutr 2010;103:1278-86.

9. Chan ES, Cummings C, Atkinson A, et al. Dietary exposures and allergy prevention in high-risk infants: a joint position statement of the Canadian Society of Allergy and Clinical Immunology and the Canadian Paediatric Society. Allergy Asthma Clin Immunol 2014;10:45.

10. Fleischer DM, Spergel JM, Assa'ad AH, et al. Primary prevention of allergic disease through nutritional interventions. J Allergy Clin Immunol Pract 2013;1:29-36.

11. Frazier AL, Camargo CA Jr, Malspeis S, et al. Prospective study of peripregnancy consumption of peanuts or tree nuts by mothers and the risk of peanut or tree nut allergy in their offspring. JAMA Pediatr 2014;168:156-62.

12. Bunyavanich S, Rifas-Shiman SL, Platts-Mills TA, et al. Peanut, milk, and wheat intake during pregnancy is associated with reduced allergy and asthma in children. J Allergy Clin Immunol 2014;133:1373-82.

13. Kramer MS, Kakuma R. Maternal dietary antigen avoidance during pregnancy or lactation, or both, or preventing or treating atopic disease in the child. Cochrane Database Syst Rev 2012;(9):CD000133.

Competing interests: Elissa Abrams reports moderator fees from Novartis. Scott Sicherer reports author and editor royalties from UpToDate, grants from the National Institute of Allergy and Infectious Diseases and HAL Allergy, and author royalties from Johns Hopkins University Press.

This article has been peer reviewed.

Affiliations: Department of Pediatrics (Abrams), Section of Allergy and Clinical Immunology, University of Manitoba, Winnipeg, Man.; Division of Allergy and Immunology (Sicherer), Icahn School of Medicine at Mount Sinai, New York, NY

Contributors: Both authors wrote the manuscript, approved the final version to be published and agreed to be accountable for all aspects of the work.

Correspondence to: Elissa Abrams, elissa.abrams@gmail.com 